\title{
Fairer Dialog gefordert
}

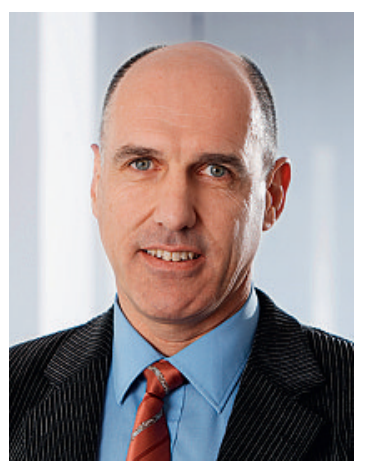

Jacques de Haller
Es führt kein Weg daran vorbei: Wir brauchen eine neue Kommunikationskultur mit einem engagierten und fairen Dialog. Nur so können wir gemeinsam mit unseren Gesundheitspartnern das schweizerische Gesundheitswesen wieder auf Kurs bringen und auch künftig eine immer noch gute medizinische Versorgung in der Schweiz sicherstellen. Zurzeit ist es jedoch um den fairen Dialog alles andere als gut bestellt.

Laut Informationen in der Sonntagspresse vom 26. April 2009 und gestützt auf ein Dokument des EDI soll sich die Verbindung der Schweizer Ärztinnen und Ärzte FMH angeblich für tiefere Medikamentenmargen ausgesprochen und bei den Labortarifen kapituliert haben. Diese Aussagen des EDI sind schlicht falsch und irreführend. Als Reaktion auf die Berichterstattung in der Sonntagspresse hatte die FMH noch

\section{«Wir brauchen eine neue Kommunikationskultur mit einem engagierten und fairen Dialog. Nur so können wir das schweizerische Gesundheitswesen wieder auf Kurs bringen»}

am selben Tag in einer Medienmitteilung (siehe S. 743) den Fehler des EDI berichtigt und sich wiederholt klipp und klar gegen tiefere Medikamentenmargen ausgesprochen. Wir bedauern, dass der Meinungsaustausch über Umwege - nämlich die Medien - stattgefunden hat.

Wenn wir in diesen beiden Fällen konstatiert hatten, es handle sich dabei um Einsparungen auf dem Buckel der Ärzteschaft, so kann und darf dies nicht als Einverständnis der Ärzteschaft gedeutet werden. Um es klar und deutlich zu sagen: Die Margen auf den Medikamenten sind nicht verhandelbar. Eine solche Massnahme ist willkürlich, entbehrt jeder wirtschaftlichen Grundlage und ist vorwiegend ein politischer Angriff, der einmal mehr die Hausärztinnen und Hausärzte in 17 Kantonen trifft. Ferner möchte ich an dieser Stelle nochmals ausdrücklich betonen, dass die FMH die Re- vision der Analysenliste, wie sie nun ab 1. Juli 2009 umgesetzt werden soll, strikt ablehnt: Die beschlossene Revision überzeugt deshalb nicht, weil sie wirtschaftlich nicht gerechtfertigt ist und die Gesundheitsversorgung der Bevölkerung grundlegend gefährdet. Als die Revision der Analysenliste in Angriff genommen wurde, hat die FMH frühzeitig und wiederholt den Austausch mit dem BAG gesucht und mit dem Modell «Point-of-Care-Tarif» einen eigenen, fairen und überzeugenden Vorschlag eingebracht. Das BAG hatte insgesamt kein Gehör für die Argumente der Ärzteschaft und zeigte sich leider alles andere als dialogbereit.

\section{«Was vonseiten der Ärzteschaft ver- handelbar ist und was nicht, müssen wir selbst bestimmen können»}

Erneut haben wir also feststellen müssen, dass eine in Bedrängnis geratene Verwaltung nicht wirklich zur Lösung der anstehenden Probleme beiträgt, mit denen wir alle konfrontiert sind. Um die notwendigen Entwicklungen durchzusetzen, braucht ein Gesundheitsminister eine politische Mehrheit oder zumindest die Unterstützung seiner eigenen Fraktion und die Fähigkeit, die betroffenen Kreise, die Stakeholder, zusammenzubringen, um die Partikularinteressen der verschiedenen Interessegruppen zu überwinden, die das Parlament seit Jahren lähmen. Wir plädieren - jetzt erst recht - für einen steten, fairen und offenen Austausch zwischen den Gesundheitspartnern und der Verwaltung. Er ist in jedem Fall weitaus erfolgversprechender als ein Alleingang. Dabei gilt jedoch: Was vonseiten der Ärzteschaft verhandelbar ist und was nicht, müssen wir selbst bestimmen können. Was für uns nicht verhandelbar ist, müssen die anderen Player akzeptieren, so wie auch wir den Verhandlungsspielraum bei unseren Partnern respektieren. Das sind die Basisregeln für einen konstruktiven Dialog. Auf dieser Prämisse aufbauend, sucht die FMH auch weiterhin den fairen Dialog mit ihren Partnern und im Besonderen mit dem Bund, um konsensfähige und überzeugende Lösungen für das Schweizer Gesundheitswesen zu finden.

Dr. med. Jacques de Haller, Präsident der FMH 